\title{
Coincidence and fixed points for contractions and cyclical contractions in partial metric spaces
}

\author{
Hassen Aydi ${ }^{1}$, Calogero Vetro ${ }^{2}$, Wutiphol Sintunavarat ${ }^{3}$ and Poom Kumam ${ }^{3 *}$
}

\section{"Correspondence:}

poom.kum@kmutt.ac.th

${ }^{3}$ Department of Mathematics,

Faculty of Science, King Mongkut's

University of Technology Thonburi (KMUTT), Bangkok, 10140, Thailand

Full list of author information is

available at the end of the article

\begin{abstract}
We prove some coincidence and common fixed point results for three mappings satisfying a generalized weak contractive condition in ordered partial metric spaces. As application of the presented results, we give a unique fixed point result for a mapping satisfying a weak cyclical contractive condition. We also provide some illustrative examples.

MSC: $47 \mathrm{H} 10 ; 54 \mathrm{H} 25$

Keywords: coincidence point; common fixed point; compatible mappings; cyclic weak $(\psi, \varphi)$-contraction; partial metric space; weakly increasing mappings
\end{abstract}

\section{Introduction and preliminaries}

In the last decades, several authors have worked on domain theory in order to equip semantics domain with a notion of distance. In 1994, Matthews [29] introduced the notion of a partial metric space as a part of the study of denotational semantics of dataflow networks and showed that the Banach contraction principle [16] can be generalized to the partial metric context for applications in program verification. Later on, many researchers studied fixed point theorems in partial metric spaces as well as ordered partial metric spaces. For more details, see $[5,6,9-15,19,20,33,34,36]$.

Recently, there have been so many exciting developments in the field of existence of fixed points in partially ordered sets. For instance, Ran and Reurings [38] extended the Banach contraction principle in partially ordered sets with some applications to matrix equations. For more details on fixed point theory in partially ordered sets, we refer the reader to [1-4, $7,8,17,18,24,28,30-32,39,41]$ and the references cited therein.

In this paper, we establish some coincidence and common fixed point results for three self-mappings on an ordered partial metric space satisfying a generalized weak contractive condition. The presented theorems extend some recent results in the literature. Moreover, as application, we give a unique fixed point theorem for a mapping satisfying a weak cyclical contractive condition.

Throughout this paper, $\mathbb{R}_{+}$will denote the set of all non-negative real numbers. First, we start by recalling some known definitions and properties of partial metric spaces.

Definition 1.1 ([29]) A partial metric on a nonempty set $X$ is a function $p: X \times X \rightarrow \mathbb{R}_{+}$ such that for all $x, y, z \in X$ :

@ 2012 Aydi et al.; licensee Springer. This is an Open Access article distributed under the terms of the Creative Commons Attribution License (http://creativecommons.org/licenses/by/2.0), which permits unrestricted use, distribution, and reproduction in any medium, provided the original work is properly cited. 
(p1) $x=y \Longleftrightarrow p(x, x)=p(x, y)=p(y, y)$,

(p2) $p(x, x) \leq p(x, y)$,

(p3) $p(x, y)=p(y, x)$,

(p4) $p(x, y) \leq p(x, z)+p(z, y)-p(z, z)$.

A partial metric space is a pair $(X, p)$ such that $X$ is a nonempty set and $p$ is a partial metric on $X$.

It is clear that, if $p(x, y)=0$, then from (p1) and (p2), $x=y$; but if $x=y, p(x, y)$ may not be 0 . A basic example of a partial metric space is the pair $\left(\mathbb{R}_{+}, p\right)$, where $p(x, y)=\max \{x, y\}$ for all $x, y \in \mathbb{R}_{+}$.

Other examples of partial metric spaces which are interesting from a computational point of view may be found in [22, 29].

Each partial metric $p$ on $X$ generates a $T_{0}$ topology $\tau_{p}$ on $X$ which has as a base the family of open $p$-balls $\left\{B_{p}(x, \varepsilon), x \in X, \varepsilon>0\right\}$, where $B_{p}(x, \varepsilon)=\{y \in X: p(x, y)<p(x, x)+\varepsilon\}$ for all $x \in X$ and $\varepsilon>0$.

If $p$ is a partial metric on $X$, then the function $p^{s}: X \times X \rightarrow \mathbb{R}_{+}$given by

$$
p^{s}(x, y)=2 p(x, y)-p(x, x)-p(y, y)
$$

is a metric on $X$.

Definition 1.2 ([29]) Let $\left\{x_{n}\right\}$ be a sequence in $X$. Then

(i) $\left\{x_{n}\right\}$ converges to a point $x \in X$ if and only if $p(x, x)=\lim _{n \rightarrow+\infty} p\left(x, x_{n}\right)$. We may write this as $x_{n} \rightarrow x$.

(ii) $\left\{x_{n}\right\}$ is called a Cauchy sequence if $\lim _{n, m \rightarrow+\infty} p\left(x_{n}, x_{m}\right)$ exists and is finite.

(iii) $(X, p)$ is said to be complete if every Cauchy sequence $\left\{x_{n}\right\}$ in $X$ converges, with respect to $\tau_{p}$, to a point $x \in X$, such that $p(x, x)=\lim _{n, m \rightarrow+\infty} p\left(x_{n}, x_{m}\right)$.

Lemma 1.3 ([29]) Let $(X, p)$ be a partial metric space. Then

(a) $\left\{x_{n}\right\}$ is a Cauchy sequence in $(X, p)$ if and only if it is a Cauchy sequence in the metric space $\left(X, p^{s}\right)$.

(b) A partial metric space $(X, p)$ is complete if and only if the metric space $\left(X, p^{s}\right)$ is complete. Furthermore, $\lim _{n \rightarrow+\infty} p^{s}\left(x_{n}, x\right)=0$ if and only if

$$
p(x, x)=\lim _{n \rightarrow+\infty} p\left(x_{n}, x\right)=\lim _{n, m \rightarrow+\infty} p\left(x_{n}, x_{m}\right)
$$

Definition 1.4 ([5]) Let $(X, p)$ be a partial metric space and $T: X \rightarrow X$ be a given mapping. We say that $T$ is continuous at $x_{0} \in X$, if for every $\varepsilon>0$, there exists $\eta>0$ such that $T\left(B_{p}\left(x_{0}, \eta\right)\right) \subseteq B_{p}\left(T x_{0}, \varepsilon\right)$.

Lemma 1.5 (Sequential characterization of continuity) Let $(X, p)$ be a partial metric space and $T: X \rightarrow X$ be a given mapping. $T: X \rightarrow X$ is continuous at $x_{0} \in X$ if it is sequentially continuous at $x_{0}$, that is, if and only if

$$
\forall\left\{x_{n}\right\} \subset X: \lim _{n \rightarrow+\infty} x_{n}=x_{0} \quad \Rightarrow \quad \lim _{n \rightarrow+\infty} T x_{n}=T x_{0} .
$$


Let $X$ be a nonempty set and $R: X \rightarrow X$ be a given mapping. For every $x \in X$, we denote by $R^{-1}(x)$ the subset of $X$ defined by

$$
R^{-1}(x):=\{u \in X \mid R u=x\} .
$$

Definition 1.6 Let $X$ be a nonempty set. Then $(X, \preceq, p)$ is called an ordered partial metric space if and only if

(i) $(X, p)$ is a partial metric space,

(ii) $(X, \preceq)$ is a partially ordered set.

Definition 1.7 Let $(X, \preceq)$ be a partially ordered set. Then $x, y \in X$ are called comparable if $x \preceq y$ or $y \preceq x$ holds.

Definition 1.8 ([30]) Let $(X, \preceq)$ be a partially ordered set and $T, S, R: X \rightarrow X$ be given mappings such that $T X \subseteq R X$ and $S X \subseteq R X$. We say that $S$ and $T$ are weakly increasing with respect to $R$ if and only if, for all $x \in X$, we have

$$
T x \preceq S y, \quad \forall y \in R^{-1}(T x)
$$

and

$$
S x \preceq T y, \quad \forall y \in R^{-1}(S x) .
$$

Remark 1.9 If $R: X \rightarrow X$ is the identity mapping ( $R x=x$ for all $x \in X$, shortly $R=I_{X}$ ), then the fact that $S$ and $T$ are weakly increasing with respect to $R$ implies that $S$ and $T$ are weakly increasing mappings, that is, $S x \preceq T S x$ and $T x \preceq S T x$ for all $x \in X$. Finally, a mapping $T: X \rightarrow X$ is weakly increasing if and only if $T x \preceq T T x$ for all $x \in X$.

Example 1.10 Consider $X=\mathbb{R}_{+}$endowed with the usual ordering of real numbers and define $T, S, R: X \rightarrow X$ by

$$
T x=3 \text { for all } x \in X, \quad S x=\left\{\begin{array}{ll}
x & \text { if } x \in[0,3], \\
3 & \text { if } x>3
\end{array} \text { and } R x=x \text { for all } x \in X .\right.
$$

Now, $R^{-1}(T x)=\{3\}$ and $R^{-1}(S x)=S x$, then $S$ and $T$ are weakly increasing with respect to $R$.

Definition 1.11 Let $(X, \preceq, p)$ be an ordered partial metric space. We say that $X$ is regular if and only if the following hypothesis holds: $\left\{z_{n}\right\}$ is a non-decreasing sequence in $X$ with respect to $\preceq$ such that $z_{n} \rightarrow z$ as $n \rightarrow+\infty$, then $z_{n} \preceq z$ for all $n \in \mathbb{N}$.

Finally, we recall the following definition of partial-compatibility introduced by Samet et al. [40].

Definition 1.12 Let $(X, p)$ be a partial metric space and $T, R: X \rightarrow X$ be given mappings. We say that the pair $\{T, R\}$ is partial-compatible if the following conditions hold:

(b1) $p(x, x)=0$ implies that $p(R x, R x)=0$. 
(b2) $\lim _{n \rightarrow+\infty} p\left(T R x_{n}, R T x_{n}\right)=0$, whenever $\left\{x_{n}\right\}$ is a sequence in $X$ such that $T x_{n} \rightarrow t$ and $R x_{n} \rightarrow t$ for some $t \in X$.

Note that Definition 1.12 extends and generalizes the notion of compatibility introduced by Jungck [25].

\section{Main results}

We start this section with some auxiliary results (see also [37]).

Lemma 2.1 Let $(X, d)$ be a metric space and let $\left\{x_{n}\right\}$ be a sequence in $X$ such that $\left\{d\left(x_{n+1}, x_{n}\right)\right\}$ is non-increasing and

$$
\lim _{n \rightarrow+\infty} d\left(x_{n+1}, x_{n}\right)=0
$$

If $\left\{x_{2 n}\right\}$ is not a Cauchy sequence, then there exist $\varepsilon>0$ and two sequences $\left\{m_{k}\right\}$ and $\left\{n_{k}\right\}$ of positive integers such that $m_{k}>n_{k}>k$ and the following four sequences tend to $\varepsilon$ when $k \rightarrow+\infty$ :

$$
\left\{d\left(x_{2 m_{k}}, x_{2 n_{k}}\right)\right\}, \quad\left\{d\left(x_{2 m_{k}}, x_{2 n_{k}+1}\right)\right\}, \quad\left\{d\left(x_{2 m_{k}-1}, x_{2 n_{k}}\right)\right\}, \quad\left\{d\left(x_{2 m_{k}-1}, x_{2 n_{k}+1}\right)\right\} .
$$

As a corollary, applying Lemma 2.1 to the associated metric $p^{s}$ of a partial metric $p$, and using Lemma 1.3, we obtain the following lemma (see also [21]).

Lemma 2.2 Let $(X, p)$ be a partial metric space and let $\left\{x_{n}\right\}$ be a sequence in $X$ such that $\left\{p\left(x_{n+1}, x_{n}\right)\right\}$ is non-increasing and

$$
\lim _{n \rightarrow+\infty} p\left(x_{n+1}, x_{n}\right)=0
$$

If $\left\{x_{2 n}\right\}$ is not a Cauchy sequence, then there exist $\varepsilon>0$ and two sequences $\left\{m_{k}\right\}$ and $\left\{n_{k}\right\}$ of positive integers such that $m_{k}>n_{k}>k$ and the following four sequences tend to $\varepsilon$ when $k \rightarrow+\infty$ :

$$
\left\{p\left(x_{2 m_{k}}, x_{2 n_{k}}\right)\right\}, \quad\left\{p\left(x_{2 m_{k}}, x_{2 n_{k}+1}\right)\right\}, \quad\left\{p\left(x_{2 m_{k}-1}, x_{2 n_{k}}\right)\right\}, \quad\left\{p\left(x_{2 m_{k}-1}, x_{2 n_{k}+1}\right)\right\} .
$$

In the sequel, let $\Psi$ be the set of functions $\psi: \mathbb{R}_{+} \rightarrow \mathbb{R}_{+}$such that $\psi$ is continuous, strictly increasing and $\psi(t)=0$ if and only if $t=0$. Also, let $\Phi$ be the set of functions $\varphi: \mathbb{R}_{+} \rightarrow \mathbb{R}_{+}$ such that $\varphi$ is lower semi-continuous and $\varphi(t)=0$ if and only if $t=0$. Such $\psi$ and $\varphi$ are called control functions.

Our first main result is the following.

Theorem 2.3 Let $(X, \preceq)$ be a partially ordered set. Suppose that there exists a partial metric $p$ on $X$ such that the partial metric space $(X, p)$ is complete. Let $T, S, R: X \rightarrow X$ be given mappings satisfying

(a) $T, S$ and $R$ are continuous,

(b) the pairs $\{R, T\}$ and $\{S, R\}$ are partial-compatible,

(c) $T$ and $S$ are weakly increasing with respect to $R$. 
Suppose that for every $(x, y) \in X \times X$ such that $R x$ and Ry are comparable, we have

$$
\psi(p(T x, S y)) \leq \psi\left(\frac{p(T x, R x)+p(S y, R y)}{2}\right)-\varphi(p(R x, R y)),
$$

where $\psi \in \Psi$ and $\varphi \in \Phi$. Then $T, S$ and $R$ have a coincidence point $u \in X$, that is, $T u=$ $\mathrm{Su}=\mathrm{Ru}$.

Proof By Definition 1.8, it follows that $T X \cup S X \subseteq R X$. Let $x_{0}$ be an arbitrary point in $X$. Since $T X \subseteq R X$, there exists $x_{1} \in X$ such that $R x_{1}=T x_{0}$. Since $S X \subseteq R X$, there exists $x_{2} \in X$ such that $R x_{2}=S x_{1}$. Continuing this process, we can construct a sequence $\left\{x_{n}\right\}$ in $X$ defined by

$$
R x_{2 n+1}=T x_{2 n}, \quad R x_{2 n+2}=S x_{2 n+1}, \quad \forall n \in \mathbb{N} .
$$

By construction, we have $x_{1} \in R^{-1}\left(T x_{0}\right)$ and $x_{2} \in R^{-1}\left(S x_{1}\right)$. Then using the fact that $S$ and $T$ are weakly increasing with respect to $R$, we obtain

$$
R x_{1}=T x_{0} \preceq S x_{1}=R x_{2} \preceq T x_{2}=R x_{3} .
$$

We continue this process to get

$$
R x_{1} \preceq R x_{2} \preceq \cdots \preceq R x_{2 n+1} \preceq R x_{2 n+2} \preceq \cdots .
$$

We claim that $\left\{R x_{n}\right\}$ is a Cauchy sequence in the partial metric space $(X, p)$. To this aim, we distinguish the following two cases.

Case 1. We suppose that there exists $k \in \mathbb{N}$ such that $p\left(R x_{2 k}, R x_{2 k+1}\right)=0$, so that $R x_{2 k}=$ $R x_{2 k+1}$. By (2.3), applying (2.1) with $x=x_{2 k}$ and $y=x_{2 k+1}$, we get

$$
\begin{aligned}
\psi\left(p\left(R x_{2 k+2}, R x_{2 k+1}\right)\right) & =\psi\left(p\left(T x_{2 k}, S x_{2 k+1}\right)\right) \\
& \leq \psi\left(\frac{p\left(T x_{2 k}, R x_{2 k}\right)+p\left(S x_{2 k+1}, R x_{2 k+1}\right)}{2}\right)-\varphi\left(p\left(R x_{2 k}, R x_{2 k+1}\right)\right) \\
& =\psi\left(\frac{p\left(R x_{2 k+1}, R x_{2 k}\right)+p\left(R x_{2 k+2}, R x_{2 k+1}\right)}{2}\right)-\varphi\left(p\left(R x_{2 k}, R x_{2 k+1}\right)\right) \\
& =\psi\left(\frac{p\left(R x_{2 k+2}, R x_{2 k+1}\right)}{2}\right) .
\end{aligned}
$$

Since $\psi$ is strictly increasing, we have

$$
p\left(R x_{2 k+2}, R x_{2 k+1}\right) \leq \frac{1}{2} p\left(R x_{2 k+2}, R x_{2 k+1}\right) .
$$

This implies that $p\left(R x_{2 k+2}, R x_{2 k+1}\right)=0$. Continuing this process, we obtain $p\left(R x_{n}, R x_{2 k}\right)=0$ for all $n \geq 2 k$. This implies that $R x_{n}=R x_{2 k}$, therefore $\left\{R x_{n}\right\}$ is Cauchy in $(X, p)$. The same conclusion holds if $R x_{2 k+1}=R x_{2 k+2}$ for some $k \in \mathbb{N}$.

Case 2. Now, we suppose that

$$
R x_{n} \neq R x_{n+1}, \quad \forall n \in \mathbb{N} .
$$


Here, we have $p\left(R x_{n}, R x_{n+1}\right) \neq 0$ for all $n \geq 0$. Thanks to (2.3), $R x_{2 n}$ and $R x_{2 n+1}$ are comparable, then using (2.2) and taking $x=x_{2 n+2}$ and $y=x_{2 n+1}$ in (2.1), we get

$$
\begin{aligned}
\psi & \left(p\left(R x_{2 n+3}, R x_{2 n+2}\right)\right) \\
& =\psi\left(p\left(T x_{2 n+2}, S x_{2 n+1}\right)\right) \\
& \leq \psi\left(\frac{p\left(T x_{2 n+2}, R x_{2 n+2}\right)+p\left(S x_{2 n+1}, R x_{2 n+1}\right)}{2}\right)-\varphi\left(p\left(R x_{2 n+2}, R x_{2 n+1}\right)\right) \\
& =\psi\left(\frac{p\left(R x_{2 n+3}, R x_{2 n+2}\right)+p\left(R x_{2 n+2}, R x_{2 n+1}\right)}{2}\right)-\varphi\left(p\left(R x_{2 n+2}, R x_{2 n+1}\right)\right) \\
& \leq \psi\left(\frac{p\left(R x_{2 n+3}, R x_{2 n+2}\right)+p\left(R x_{2 n+2}, R x_{2 n+1}\right)}{2}\right) .
\end{aligned}
$$

Since $\psi$ is strictly increasing, the above inequality implies that

$$
p\left(R x_{2 n+3}, R x_{2 n+2}\right) \leq p\left(R x_{2 n+2}, R x_{2 n+1}\right) .
$$

Now, taking $x=x_{2 n}$ and $y=x_{2 n+1}$ in (2.1), we have

$$
\begin{aligned}
\psi & \left(p\left(R x_{2 n+1}, R x_{2 n+2}\right)\right) \\
& =\psi\left(p\left(T x_{2 n}, S x_{2 n+1}\right)\right) \\
& \leq \psi\left(\frac{p\left(T x_{2 n}, R x_{2 n}\right)+p\left(S x_{2 n+1}, R x_{2 n+1}\right)}{2}\right)-\varphi\left(p\left(R x_{2 n}, R x_{2 n+1}\right)\right) \\
& =\psi\left(\frac{p\left(R x_{2 n+1}, R x_{2 n}\right)+p\left(R x_{2 n+2}, R x_{2 n+1}\right)}{2}\right)-\varphi\left(p\left(R x_{2 n}, R x_{2 n+1}\right)\right) \\
& \leq \psi\left(\frac{p\left(R x_{2 n+1}, R x_{2 n}\right)+p\left(R x_{2 n+2}, R x_{2 n+1}\right)}{2}\right),
\end{aligned}
$$

which implies that

$$
p\left(R x_{2 n+2}, R x_{2 n+1}\right) \leq p\left(R x_{2 n+1}, R x_{2 n}\right) .
$$

Combining (2.5) and (2.7), we get

$$
p\left(R x_{n+1}, R x_{n+2}\right) \leq p\left(R x_{n}, R x_{n+1}\right) \quad \text { for all } n \geq 0 .
$$

It follows that the sequence $\left\{p\left(R x_{n}, R x_{n+1}\right)\right\}$ is non-increasing and bounded below by 0 . Hence, there exists $r \geq 0$ such that

$$
p\left(R x_{n}, R x_{n+1}\right) \rightarrow r \quad \text { as } n \rightarrow+\infty .
$$

We claim that $r=0$. Suppose that $r>0$. Taking the lim sup as $n \rightarrow+\infty$ in (2.6) and using the properties of the functions $\psi$ and $\varphi$, we have

$$
\psi(r) \leq \psi(r)-\varphi(r) .
$$


This implies that $\varphi(r)=0$, and by a property of the function $\varphi$, we have $r=0$, that is a contradiction. We deduce that $r=0$, i.e.,

$$
p\left(R x_{n}, R x_{n+1}\right) \rightarrow 0 \quad \text { as } n \rightarrow+\infty .
$$

We shall show that $\left\{R x_{n}\right\}$ is a Cauchy sequence in the partial metric space $(X, p)$. For this, it is sufficient to prove that $\left\{R x_{2 n}\right\}$ is Cauchy in $(X, p)$. Suppose to the contrary that $\left\{R x_{2 n}\right\}$ is not a Cauchy sequence. Then, having in mind that $\left\{p\left(R x_{n}, R x_{n+1}\right)\right\}$ is non-increasing and (2.9), it follows by Lemma 2.2 that there exist $\varepsilon>0$ and two sequences $\left\{m_{k}\right\}$ and $\left\{n_{k}\right\}$ of positive integers such that $m_{k}>n_{k}>k$ and the following four sequences tend to $\varepsilon$ when $k \rightarrow+\infty$ :

$$
\begin{array}{ll}
\left\{p\left(R x_{2 m_{k}}, R x_{2 n_{k}}\right)\right\}, & \left\{p\left(R x_{2 m_{k}}, R x_{2 n_{k}+1}\right)\right\}, \\
\left\{p\left(R x_{2 m_{k}-1}, R x_{2 n_{k}}\right)\right\}, & \left\{p\left(R x_{2 m_{k}-1}, R x_{2 n_{k}+1}\right)\right\} .
\end{array}
$$

Applying (2.1) with $x=x_{2 n_{k}}$ and $y=x_{2 m_{k}-1}$, we get

$$
\begin{aligned}
\psi & \left(p\left(R x_{2 n_{k}+1}, R x_{2 m_{k}}\right)\right) \\
& =\psi\left(p\left(T x_{2 n_{k}}, S x_{2 m_{k}-1}\right)\right) \\
& \leq \psi\left(\frac{p\left(T x_{2 n_{k}}, R x_{2 n_{k}}\right)+p\left(S x_{2 m_{k}-1}, R x_{2 m_{k}-1}\right)}{2}\right)-\varphi\left(p\left(R x_{2 n_{k}}, R x_{2 m_{k}-1}\right)\right) \\
& =\psi\left(\frac{p\left(R x_{2 n_{k}+1}, R x_{2 n(k)}\right)+p\left(R x_{2 m(k)}, R x_{2 m_{k}-1}\right)}{2}\right)-\varphi\left(p\left(R x_{2 n_{k}}, R x_{2 m_{k}-1}\right)\right) .
\end{aligned}
$$

Taking lim $\sup _{k \rightarrow+\infty}$ in the above inequality and using the continuity of $\psi$ and the lower semi-continuity of $\varphi$, we obtain

$$
\psi(\varepsilon) \leq \psi\left(\frac{0+0}{2}\right)-\varphi(\varepsilon)=-\varphi(\varepsilon)
$$

from which a contradiction follows since $\varepsilon>0$. Then, we deduce that $\left\{R x_{n}\right\}$ is a Cauchy sequence in the partial metric space $(X, p)$, which is complete, so $\left\{R x_{n}\right\}$ converges to some $u \in X$, that is, from (p3) and Definition 1.2,

$$
p(u, u)=\lim _{n \rightarrow+\infty} p\left(R x_{n}, u\right)=\lim _{n \rightarrow+\infty} p\left(R x_{n}, R x_{n}\right) .
$$

But from (2.9) and condition (p2), we have

$$
\lim _{n \rightarrow+\infty} p\left(R x_{n}, R x_{n}\right)=0
$$

therefore, it follows that

$$
p(u, u)=\lim _{n \rightarrow+\infty} p\left(R x_{n}, u\right)=\lim _{n \rightarrow+\infty} p\left(R x_{n}, R x_{n}\right)=0 .
$$

From (2.11) and the continuity of $R$, we get

$$
\lim _{n \rightarrow+\infty} p\left(R\left(R x_{n}\right), R u\right)=p(R u, R u) .
$$


The triangular inequality yields

$$
p(R u, T u) \leq p\left(R u, R\left(R x_{2 n+1}\right)\right)+p\left(R\left(T x_{2 n}\right), T\left(R x_{2 n}\right)\right)+p\left(T\left(R x_{2 n}\right), T u\right)
$$

By (2.2) and (2.11), we have

$$
R x_{2 n} \rightarrow u, \quad T x_{2 n}=R x_{2 n+1} \rightarrow u \quad \text { as } n \rightarrow+\infty
$$

Having in mind that the pair $\{R, T\}$ is partial-compatible, then

$$
p\left(R\left(T x_{2 n}\right), T\left(R x_{2 n}\right)\right) \rightarrow 0 \text { as } n \rightarrow+\infty .
$$

Also, since $p(u, u)=0$, then we have $p(T u, T u)=0$. The continuity of $T$ together with (2.11) give us

$$
p\left(T\left(R x_{2 n}\right), T u\right) \rightarrow p(T u, T u)=0 .
$$

Combining (2.12) and (2.15) together with (2.16) and letting $n \rightarrow+\infty$ in (2.13), we obtain

$$
p(R u, T u) \leq p(R u, R u)
$$

By condition (p2) and (2.17), one can write

$$
p(R u, R u)=p(R u, T u) .
$$

Similarly, by triangular inequality, we get

$$
p(R u, S u) \leq p\left(R u, R\left(R x_{2 n+2}\right)\right)+p\left(R\left(S x_{2 n+1}\right), S\left(R x_{2 n+1}\right)\right)+p\left(S\left(R x_{2 n+1}\right), S u\right) .
$$

By (2.2) and (2.11), we have

$$
R x_{2 n+1} \rightarrow u, \quad S x_{2 n+1} \rightarrow u \quad \text { as } n \rightarrow+\infty .
$$

Since the pair $\{S, R\}$ is partial-compatible, then

$$
p\left(R\left(S x_{2 n+1}\right), S\left(R x_{2 n+1}\right)\right) \rightarrow 0 \quad \text { as } n \rightarrow+\infty .
$$

Also, since $p(u, u)=0$, it follows $p(R u, R u)=0$. Thus, from $(2.18), p(R u, T u)=p(R u, R u)=0$ and so $R u=T u$.

The continuity of $S$ and (2.20) give us

$$
p\left(S\left(R x_{2 n+1}\right), S u\right) \rightarrow p(S u, S u) \quad \text { as } n \rightarrow+\infty .
$$

Combining (2.12) and (2.21) together with (2.22) and letting $n \rightarrow+\infty$ in (2.19), we obtain

$$
p(R u, S u) \leq p(R u, R u)+p(S u, S u)=p(S u, S u) .
$$


By condition (p2) and (2.23), we get

$$
p(R u, S u)=p(S u, S u)
$$

Applying (2.1) with $x=y=u$, we get

$$
\begin{aligned}
\psi(p(T u, S u)) & \leq \psi\left(\frac{p(T u, R u)+p(S u, R u)}{2}\right)-\varphi(p(R u, R u)) \\
& =\psi\left(\frac{p(S u, T u)}{2}\right)-\varphi(0) \\
& =\psi\left(\frac{p(T u, S u)}{2}\right)
\end{aligned}
$$

This implies that

$$
p(T u, S u) \leq \frac{1}{2} p(T u, S u)
$$

and so it follows $p(T u, S u)=0$, that is $T u=S u$. Thus, we have obtained

$$
R u=T u=S u,
$$

that is, $u$ is a coincidence point of $T, S$ and $R$.

Remark 2.4 We point out that the order in which the mappings in condition (b) of Theorem 2.3 are considered is crucial. Trivially, Theorem 2.3 remains true if we assume that the partial-compatible pairs are $\{T, R\}$ and $\{R, S\}$.

Example 2.5 Let $X=\left[0, \frac{1}{2}\right]$ be endowed with the partial metric $p(x, y)=\max \{x, y\}$ and the order given as follows:

$$
x \preceq y \quad \Longleftrightarrow \quad x \geq y \text {. }
$$

Consider the mappings $T, S, R: X \rightarrow X$ defined by $T x=S x=x^{4}$ and $R x=x$ for all $x \in X$. Also, define the functions $\psi, \varphi: \mathbb{R}_{+} \rightarrow \mathbb{R}_{+}$by $\psi(t)=t$ and $\varphi(t)=\frac{t}{4}$, for all $t \geq 0$. Clearly, condition (2.1) is satisfied. In fact, for every $(x, y) \in X \times X$ with $x \geq y$, we get

$$
\begin{aligned}
\psi(p(T x, S y)) & =x^{4} \leq \frac{x}{4} \leq \frac{x+y}{2}-\frac{x}{4} \\
& =\psi\left(\frac{p(T x, R x)+p(S y, R y)}{2}\right)-\varphi(p(R x, R y))
\end{aligned}
$$

All the other hypotheses of Theorem 2.3 are satisfied and $T, S$ and $R$ have a coincidence point $u=0$. (Moreover, $u=0$ is the unique common fixed point of $T, S$ and $R$.)

Note that Theorem 2.3 is not applicable in respect of the usual order of real numbers because $T$ is not weakly increasing. It follows that the partial order may be fundamental.

Under different hypotheses, the conclusion of Theorem 2.3 remains true without assuming the continuity of $T, S$ and $R$, and the partial-compatibility of the pairs $\{T, R\}$ and $\{R, S\}$. This is the purpose of the next theorem. 
Theorem 2.6 Let $(X, \preceq)$ be a partially ordered set. Suppose that there exists a partial metric $p$ on $X$ such that $(X, p)$ is complete. Let $T, S, R: X \rightarrow X$ be given mappings satisfying

(a) $R X$ is a closed subspace of $(X, p)$,

(b) $T$ and $S$ are weakly increasing with respect to $R$,

(c) $X$ is regular.

Suppose that for every $(x, y) \in X \times X$ such that $R x$ and Ry are comparable, we have

$$
\psi(p(T x, S y)) \leq \psi\left(\frac{p(T x, R x)+p(S y, R y)}{2}\right)-\varphi(p(R x, R y)),
$$

where $\psi \in \Psi$ and $\varphi \in \Phi$. Then, $T, S$ and $R$ have a coincidence point $u \in X$, that is, Tu= $\mathrm{Su}=\mathrm{Ru}$.

Proof Following the proof of Theorem 2.3, we have that $\left\{R x_{n}\right\}$ is a Cauchy sequence in the closed subspace $R X$, then there exists $v=R u$, with $u \in X$, such that

$$
R x_{n} \rightarrow v=R u \quad \text { as } n \rightarrow+\infty .
$$

Thanks to (2.3), $\left\{R x_{n}\right\}$ is a non-decreasing sequence, and so, since it converges to $v=R u$, from the regularity of $X$, we get

$$
R x_{n} \preceq R u, \quad \forall n \in \mathbb{N} .
$$

Therefore, $R x_{n}$ and $R u$ are comparable. Putting $x=x_{2 n}$ and $y=u$ in (2.25) and using (2.2), we get

$$
\begin{aligned}
\psi & \left(p\left(R x_{2 n+1}, S u\right)\right) \\
& =\psi\left(p\left(T x_{2 n}, S u\right)\right) \\
& \leq \psi\left(\frac{p\left(T x_{2 n}, R x_{2 n}\right)+p(S u, R u)}{2}\right)-\varphi\left(p\left(R x_{2 n}, R u\right)\right) \\
& =\psi\left(\frac{p\left(R x_{2 n+1}, R x_{2 n}\right)+p(S u, R u)}{2}\right)-\varphi\left(p\left(R x_{2 n}, R u\right)\right) .
\end{aligned}
$$

Taking $\lim \sup _{n \rightarrow+\infty}$ in the above inequality, using (2.26) and the properties of $\varphi$ and $\psi$, we obtain

$$
\begin{aligned}
\psi(p(R u, S u)) & \leq \psi\left(\frac{p(S u, R u)}{2}\right)-\varphi(p(R u, R u)) \\
& \leq \psi\left(\frac{p(S u, R u)}{2}\right) .
\end{aligned}
$$

This implies that

$$
p(R u, S u) \leq \frac{1}{2} p(S u, R u),
$$

which is true if $p(S u, R u)=0$. This means that $S u=R u$. 
Analogously, putting $x=u$ and $y=x_{2 n+1}$ in (2.25), we have

$$
\begin{aligned}
\psi\left(p\left(T u, R x_{2 n+2}\right)\right. & =\psi\left(p\left(T u, S x_{2 n+1}\right)\right) \\
& \leq \psi\left(\frac{p(T u, R u)+p\left(S x_{2 n+1}, R x_{2 n+1}\right)}{2}\right)-\varphi\left(p\left(R u, R x_{2 n+1}\right)\right) \\
& =\psi\left(\frac{p\left(R x_{2 n+2}, R x_{2 n+1}\right)+p(T u, R u)}{2}\right)-\varphi\left(p\left(R u, R x_{2 n+1}\right)\right) .
\end{aligned}
$$

Taking $\lim \sup _{n \rightarrow+\infty}$ in the above inequality, using (2.26) and the properties of $\varphi$ and $\psi$, we obtain

$$
p(T u, R u) \leq \frac{1}{2} p(T u, R u)
$$

which yields that

$$
T u=R u \text {. }
$$

We conclude that $u$ is a coincidence point of $T, S$ and $R$.

If $R: X \rightarrow X$ is the identity mapping $I_{X}$, by Theorem 2.6, we obtain the following common fixed point result involving two mappings.

Corollary 2.7 Let $(X, \preceq)$ be a partially ordered set. Suppose that there exists a partial metric $p$ on $X$ such that the partial metric space $(X, p)$ is complete. Let $X$ be regular and $T, S: X \rightarrow X$ be given mappings such that $T$ and $S$ are weakly increasing. Suppose that for every $(x, y) \in X \times X$ such that $x$ and $y$ are comparable, we have

$$
\psi(p(T x, S y)) \leq \psi\left(\frac{p(T x, x)+p(S y, y)}{2}\right)-\varphi(p(x, y))
$$

where $\psi \in \Psi$ and $\varphi \in \Phi$. Then, $T$ and $S$ have a common fixed point $u \in X$, that is, $T u=$ $S u=u$.

The following example shows that the hypothesis ' $T$ and $S$ are weakly increasing (with respect to $R$ )' has a key role for the validity of our results.

Example 2.8 Let $X=[0,1]$ be endowed with the partial metric $p(x, y)=\max \{x, y\}$ and the order $\preceq$ given as follows:

$$
x \preceq y \quad \Longleftrightarrow \quad x \geq y \text {. }
$$

Consider the mappings $T, S: X \rightarrow X$ defined by $T x=\frac{x}{4}$ and $S x=\frac{x}{3}$, for all $x \in X$. Also, define the functions $\psi, \varphi: \mathbb{R}_{+} \rightarrow \mathbb{R}_{+}$by $\psi(t)=t$ and $\varphi(t)=\frac{t}{7}$, for all $t \geq 0$. It is easy to show that $S x \preceq T S x$ and $T x \preceq S T x$, for all $x \in X$, that is, $T$ and $S$ are weakly increasing. Now, take $x$ and $y$ comparable and, without loss of generality, assume $y \preceq x$, so that $x \leq y$. It is easy to show that (2.27) holds and all the other hypotheses of Corollary 2.7 are satisfied. Then, $T$ and $S$ have a unique common fixed point $u=0$. 
Note that Corollary 2.7 is not applicable in respect of the usual order of real numbers because $T$ and $S$ are not weakly increasing.

Now, we shall prove the existence and uniqueness of a common fixed point for three mappings.

Theorem 2.9 In addition to the hypotheses of Theorem 2.3, suppose that for any $(x, y) \in$ $X \times X$, there exists $z \in X$ such that $T x \preceq T z$ and $T y \preceq T z$. Then, $T, S$ and $R$ have a unique common fixed point, that is, there exists a unique $u \in X$ such that $u=R u=T u=S u$.

Proof Referring to Theorem 2.3, the set of coincidence points of $T, S$ and $R$ is nonempty. Now, we shall show that if $x^{*}$ and $y^{*}$ are coincidence points of $T, S$ and $R$, that is, $R x^{*}=$ $T x^{*}=S x^{*}$ and $R y^{*}=T y^{*}=S y^{*}$, then

$$
p\left(R x^{*}, R y^{*}\right)=0 .
$$

For the coincidence points $x^{*}$ and $y^{*}$, Theorem 2.3 gives us that

$$
p\left(R x^{*}, T x^{*}\right)=p\left(T x^{*}, S x^{*}\right)=0=p\left(R y^{*}, T y^{*}\right)=p\left(T y^{*}, S y^{*}\right) .
$$

By assumption, there exists $z_{0} \in X$ such that

$$
T x^{*} \preceq T z_{0}, \quad T y^{*} \preceq T z_{0} .
$$

Now, proceeding similarly to the proof of Theorem 2.3 , we can immediately define a sequence $\left\{R z_{n}\right\}$ as follows:

$$
R z_{2 n+1}=T z_{2 n}, \quad R z_{2 n+2}=S z_{2 n+1}, \quad \forall n \in \mathbb{N} .
$$

Since $T$ and $S$ are weakly increasing with respect to $R$, we have

$$
T x^{*}=R x^{*} \preceq R z_{n}, \quad T y^{*}=R y^{*} \preceq R z_{n}, \quad \forall n \in \mathbb{N} .
$$

Putting $x=z_{2 n}$ and $y=x^{*}$ in (2.1) and using (2.31), we get

$$
\begin{aligned}
\psi\left(p\left(R z_{2 n+1}, R x^{*}\right)\right) & =\psi\left(p\left(T z_{2 n}, S x^{*}\right)\right) \\
& \leq \psi\left(\frac{p\left(T z_{2 n}, R z_{2 n}\right)+p\left(S x^{*}, R x^{*}\right)}{2}\right)-\varphi\left(p\left(R z_{2 n}, R x^{*}\right)\right) \\
& =\psi\left(\frac{p\left(R z_{2 n+1}, R z_{2 n}\right)}{2}\right)-\varphi\left(p\left(R z_{2 n}, R x^{*}\right)\right) \\
& \leq \psi\left(\frac{p\left(R z_{2 n+1}, R z_{2 n}\right)}{2}\right) .
\end{aligned}
$$

Since $\psi$ is strictly increasing, we have

$$
p\left(R z_{2 n+1}, R x^{*}\right) \leq \frac{1}{2} p\left(R z_{2 n+1}, R z_{2 n}\right) \leq \frac{1}{2} p\left(R z_{2 n+1}, R x^{*}\right)+\frac{1}{2} p\left(R x^{*}, R z_{2 n}\right) .
$$


This gives us

$$
p\left(R z_{2 n+1}, R x^{*}\right) \leq p\left(R z_{2 n}, R x^{*}\right) .
$$

Putting $x=x^{*}$ and $y=z_{2 n}$ in (2.1), then similarly to the above, one can find

$$
p\left(R z_{2 n+2}, R x^{*}\right) \leq p\left(R z_{2 n+1}, R x^{*}\right) .
$$

We combine (2.32) and (2.33) to remark that

$$
p\left(R z_{n+1}, R x^{*}\right) \leq p\left(R z_{n}, R x^{*}\right), \quad \forall n \in \mathbb{N} \text {. }
$$

Then, the sequence $\left\{p\left(R z_{n}, R x^{*}\right)\right\}$ is non-increasing and bounded below, so there exists $r \geq 0$ such that

$$
p\left(R z_{n}, R x^{*}\right) \rightarrow r \quad \text { as } n \rightarrow+\infty \text {. }
$$

Adopting the strategy used in the proof of Theorem 2.3, one can show that $r=0$, i.e.,

$$
p\left(R z_{n}, R x^{*}\right) \rightarrow 0 \quad \text { as } n \rightarrow+\infty
$$

The same idea yields

$$
p\left(R z_{n}, R y^{*}\right) \rightarrow 0 \quad \text { as } n \rightarrow+\infty
$$

Now, $p\left(R x^{*}, R y^{*}\right) \leq p\left(R x^{*}, R z_{n}\right)+p\left(R z_{n}, R y^{*}\right)$ and from (2.35), (2.36), we obtain $p\left(R x^{*}, R y^{*}\right)=$ 0 , and so (2.28) holds.

Thanks to (2.30) and (2.35), one can write

$$
T z_{2 n} \rightarrow R x^{*}=R y^{*}, \quad S z_{2 n+1} \rightarrow R x^{*}=R y^{*} \quad \text { as } n \rightarrow+\infty .
$$

From partial-compatibility of the pairs $\{R, T\}$ and $\{S, R\}$, using (2.35) and (2.37), we obtain

$$
p\left(R\left(T z_{2 n}\right), T\left(R z_{2 n}\right)\right) \rightarrow 0, \quad p\left(R\left(S z_{2 n+1}\right), S\left(R z_{2 n+1}\right)\right) \rightarrow 0 \quad \text { as } n \rightarrow+\infty .
$$

Denote

$$
u=R x^{*}
$$

Since $p(u, u)=p\left(R x^{*}, R y^{*}\right)=0$, so again by partial-compatibility of the pairs $\{R, T\}$ and $\{S, R\}$, we get

$$
p(T u, T u)=p(R u, R u)=0 .
$$

By triangular inequality, we have

$$
p(R u, T u) \leq p\left(R u, R\left(T z_{2 n}\right)\right)+p\left(R\left(T z_{2 n}\right), T\left(R z_{2 n}\right)\right)+p\left(T\left(R z_{2 n}\right), T u\right) .
$$


Using (2.37), (2.38), (2.39), the continuity of $T$ and letting $n \rightarrow+\infty$ in the above inequality, we get

$$
p(R u, T u) \leq p(R u, R u)+p(T u, T u)=0,
$$

that is, $R u=T u$ and $u$ is a coincidence point of $T$ and $R$.

Analogously, the triangular inequality gives us

$$
p(R u, S u) \leq p\left(R u, R\left(S z_{2 n+1}\right)\right)+p\left(R\left(S z_{2 n+1}\right), S\left(R z_{2 n+1}\right)\right)+p\left(S\left(R z_{2 n+1}\right), S u\right) .
$$

Using (2.37), (2.38), (2.39), the continuity of $S$ and letting $n \rightarrow+\infty$ in the above inequality, we get

$$
p(R u, S u) \leq p(R u, R u)+p(S u, S u)=p(S u, S u) .
$$

By condition ( $\mathrm{p} 2)$, it follows immediately

$$
p(R u, S u)=p(S u, S u) .
$$

Now, applying (2.1) with $x=y=u$, we have

$$
\begin{aligned}
\psi(p(T u, S u)) & \leq \psi\left(\frac{p(T u, R u)+p(S u, R u)}{2}\right)-\varphi(p(R u, R u)) \\
& =\psi\left(\frac{p(S u, T u)}{2}\right)-\varphi(0) \\
& =\psi\left(\frac{p(T u, S u)}{2}\right) .
\end{aligned}
$$

This implies that

$$
p(T u, S u) \leq \frac{1}{2} p(T u, S u)
$$

then we deduce that $p(T u, S u)=0$, and so $T u=S u$. Until now, we have obtained

$$
R u=T u=S u .
$$

With $y^{*}=u$ and from (2.28), we have

$$
u=R x^{*}=R u=T u=S u .
$$

This proves that $u$ is a common fixed point of the mappings $T, S$ and $R$.

Now our purpose is to check that such a point is unique. Suppose to the contrary that there is another common fixed point of $T, S$ and $R$, say $q$. Then, applying (2.1) with $x=$ $y=q$, we obtain easily that $p(q, T q)=p(q, S q)=p(q, R q)=0$. It is immediate that $q$ is a coincidence point of $T, S$ and $R$. From (2.28), this implies that

$$
R q=R u
$$


Hence, we get

$$
q=R q=R u=u
$$

which yields the uniqueness of the common fixed point of $T, S$ and $R$. This completes the proof.

Remark 2.10 We leave, as exercise for the reader, to verify that our results hold even if we replace condition (2.1) by the following

$$
\psi(p(T x, S y)) \leq \psi\left(\frac{p(T x, R y)+p(S y, R x)}{2}\right)-\varphi(p(R x, R y))
$$

for all $x, y \in X$ such that $R x$ and $R y$ are comparable.

\section{Application to cyclical contractions}

In this section we use the previous results to prove a fixed point theorem for a mapping satisfying a weak cyclical contractive condition. In 2003, Kirk et al. [27] studied existence and uniqueness of a fixed point for mappings satisfying cyclical contractive conditions in complete metric spaces.

Definition 3.1 Let $(X, d)$ be a metric space, $m$ a positive integer and $Y_{1}, \ldots, Y_{m}$ nonempty subsets of $X$. A mapping $T$ on $\bigcup_{i=1}^{m} Y_{i}$ is called a $m$-cyclic mapping if $T\left(Y_{i}\right) \subset Y_{i+1}, i=$ $1, \ldots, m$, where $Y_{m+1}=Y_{1}$.

Later on, Pacurar and Rus [35] introduced the following notion, suggested by the considerations in [27].

Definition 3.2 Let $Y$ be a nonempty set, $m$ a positive integer and $T: Y \rightarrow Y$ an operator. By definition, $Y=\bigcup_{i=1}^{m} Y_{i}$ is a cyclic representation of $Y$ with respect to $T$ if $T$ is a $m$-cyclic mapping and $Y_{i}$ are nonempty sets.

Example 3.3 Let $X=\mathbb{R}$. Assume $Y_{1}=Y_{3}=[-2,0]$ and $Y_{2}=Y_{4}=[0,2]$, so that $Y=$ $\bigcup_{i=1}^{4} Y_{i}=[-2,2]$. Define $T: Y \rightarrow Y$ such that $T x=-\frac{x}{2}$, for all $x \in Y$. It is clear that $Y=\bigcup_{i=1}^{4} Y_{i}$ is a cyclic representation of $Y$.

Inspired by Karapinar [26] and Gopal et al. [23], we present the notion of a cyclic weak $(\psi, \varphi)$-contraction in partial metric spaces.

Definition 3.4 Let $(X, \preceq, p)$ be an ordered partial metric space, $Y_{1}, Y_{2}, \ldots, Y_{m}$ be closed subsets of $X$ and $Y=\bigcup_{i=1}^{m} Y_{i}$. An operator $T: Y \rightarrow Y$ is called a cyclic weak $(\psi, \varphi)$ contraction if the following conditions hold:

(i) $Y=\bigcup_{i=1}^{m} Y_{i}$ is a cyclic representation of $Y$ with respect to $T$,

(ii) there exist $\psi \in \Psi$ and $\varphi \in \Phi$ such that

$$
\psi(p(T x, T y)) \leq \psi\left(\frac{p(T x, x)+p(T y, y)}{2}\right)-\varphi(p(x, y)),
$$

for every comparable $x \in Y_{i}, y \in Y_{i+1}(i=1,2, \ldots, m)$. 
Now, we state and prove the following result.

Theorem 3.5 Let $(X, \preceq)$ be a partially ordered set. Suppose that there exists a partial metric $p$ on $X$ such that the partial metric space $(X, p)$ is complete. Let $T: \bigcup_{i=1}^{m} Y_{i} \rightarrow \bigcup_{i=1}^{m} Y_{i}$ be a given mapping satisfying

(a) $T$ is a cyclic weak $(\psi, \varphi)$-contraction,

(b) $T$ is weakly increasing and continuous,

(c) the pair $\left\{I_{x}, T\right\}$ is partial-compatible,

(d) for any $(x, y) \in X \times X$, there exists $z \in X$ such that $T x \preceq T z$ and $T y \preceq T z$.

Then, $T$ has a unique fixed point $u \in \bigcap_{i=1}^{m} Y_{i}$, that is, $T u=u$.

Proof Let $x_{0} \in Y=\bigcup_{i=1}^{m} Y_{i}$ and set

$$
x_{n+1}=T x_{n}, \quad \forall n \in \mathbb{N} .
$$

For any $n \in \mathbb{N}$, there is $i_{n} \in\{1, \ldots, m\}$ such that $x_{n} \in Y_{i_{n}}$ and $x_{n+1} \in Y_{i_{n}+1}$. Then, following the lines of the proof of Theorem 2.3, it is easy to show that $\left\{x_{n}\right\}$ is a Cauchy sequence in the partial metric space $(Y, p)$, which is complete, so $\left\{x_{n}\right\}$ converges to some $y \in Y$. On the other hand, by condition (i) of Definition 3.4, it follows that the iterative sequence $\left\{x_{n}\right\}$ has an infinite number of terms in $Y_{i}$ for each $i=1,2, \ldots, m$. Since $(Y, p)$ is complete, from each $Y_{i}, i=1,2, \ldots, m$, one can extract a subsequence of $\left\{x_{n}\right\}$ that converges to $y$. In virtue of the fact that each $Y_{i}, i=1,2, \ldots, m$, is closed, we conclude that $y \in \bigcap_{i=1}^{m} Y_{i}$ and thus $\bigcap_{i=1}^{m} Y_{i} \neq \emptyset$. Obviously, $\bigcap_{i=1}^{m} Y_{i}$ is closed and complete. Now, consider the restriction of $T$ on $\bigcap_{i=1}^{m} Y_{i}$, that is $T \mid \bigcap_{i=1}^{m} Y_{i}: \bigcap_{i=1}^{m} Y_{i} \rightarrow \bigcap_{i=1}^{m} Y_{i}$ which satisfies the assumptions of Theorem 2.3 and thus, $T \mid \bigcap_{i=1}^{m} Y_{i}$ has a unique fixed point in $\bigcap_{i=1}^{m} Y_{i}$, say $u$, which is obtained by iteration from the starting point $x_{0} \in Y$. To conclude, we have to show that, for any initial value $x \in Y$, we get the same limit point $u \in \bigcap_{i=1}^{m} Y_{i}$. Due to condition (c) and using the analogous ideas of the proof of Theorem 2.9, it can be obtained that, for any initial value $x \in Y, x_{n} \rightarrow u$ as $n \rightarrow+\infty$. This completes the proof.

Competing interests

The authors declare that they have no competing interests.

Authors' contributions

All authors contributed equally and significantly to writing this paper. All authors read and approved the final manuscript.

\section{Author details}

${ }^{1}$ Institut Supérieur d'Informatique et des Technologies de Communication de Hammam Sousse, Université de Sousse, Route GP1-4011, H. Sousse, Tunisia. ${ }^{2}$ Dipartimento di Matematica e Informatica, Università degli Studi di Palermo, Via Archirafi 34, Palermo, 90123, Italy. ${ }^{3}$ Department of Mathematics, Faculty of Science, King Mongkut's University of Technology Thonburi (KMUTT), Bangkok, 10140, Thailand.

\section{Acknowledgements}

The authors are really thankful to the anonymous referee for his/her precious suggestions useful to improve the quality of the paper. The third author would like to thank the Research Professional Development Project under the Science Achievement Scholarship of Thailand (SAST) and the forth author would like to thank the Commission on Higher Education and the Thailand Research Fund under Grant MRG no. 5380044 for financial support during the preparation of this manuscript. 


\section{References}

1. Abbas, M, Nazir, T, Radenović, S: Common fixed points of four maps in partially ordered metric spaces. Appl. Math Lett. 24, 1520-1526 (2011)

2. Abbas, M, Sintunavarat, W, Kumam, P: Coupled fixed point in partially ordered G-metric spaces. Fixed Point Theory Appl. (to appear)

3. Agarwal, RP, El-Gebeily, MA, O'Regan, D: Generalized contractions in partially ordered metric spaces. Appl. Anal. 87, 109-116 (2008)

4. Altun, I, Simsek, H: Some fixed point theorems on ordered metric spaces and application. Fixed Point Theory Appl. 2010, Article ID 621469 (2010)

5. Altun, I, Erduran, A: Fixed point theorems for monotone mappings on partial metric spaces. Fixed Point Theory Appl. 2011, Article ID 508730 (2011)

6. Altun, I, Sola, F, Simsek, H: Generalized contractions on partial metric spaces. Topol. Appl. 157(18), 2778-2785 (2010)

7. Aydi, $\mathrm{H}$ : Coincidence and common fixed point results for contraction type maps in partially ordered metric spaces. Int. J. Math. Anal. 5(3), 631-642 (2011)

8. Aydi, H, Nashine, HK, Samet, B, Yazidi, H: Coincidence and common fixed point results in partially ordered cone metric spaces and applications to integral equations. Nonlinear Anal. 74(17), 6814-6825 (2011)

9. Aydi, H: Some coupled fixed point results on partial metric spaces. Int. J. Math. Math. Sci. 2011, Article ID 64709 (2011)

10. Aydi, H: Some fixed point results in ordered partial metric. J. Nonlinear Sci. Appl. 4(3), 210-217 (2011)

11. Aydi, H: Fixed point results for weakly contractive mappings in ordered partial metric spaces. J. Adv. Math. Stud. 4(2), 1-12 (2011)

12. Aydi, H: Fixed point theorems for generalized weakly contractive condition in ordered partial metric spaces. J. Nonlinear Analysis Optim. 2(2), 33-48 (2011)

13. Aydi, $\mathrm{H}$ : Common fixed point results for mappings satisfying $(\psi, \phi)$-weak contractions in ordered partial metric spaces. Int. J. Math. Stat. 12(2), 53-64 (2012)

14. Aydi, H: A common fixed point result by altering distances involving a contractive condition of integral type in partial metric spaces. Demonstr. Math. 46(1/2) (2013) (in press)

15. Aydi, $\mathrm{H}$, Karapınar, E, Shatanawi, W: Coupled fixed point results for $(\psi, \varphi)$-weakly contractive condition in ordered partial metric spaces. Comput. Math. Appl. 62, 4449-4460 (2011)

16. Banach, S: Sur les opérations dans les ensembles abstraits et leur application aux équations intgérales. Fundam. Math. 3, 133-181 (1922)

17. Bhashkar, TG, Lakshmikantham, V: Fixed point theorems in partially ordered cone metric spaces and applications. Nonlinear Anal. 65(7), 825-832 (2006)

18. Ćirić, LB, Cakić, N, Rajović, M, Ume, JS: Monotone generalized nonlinear contractions in partially ordered metric spaces. Fixed Point Theory Appl. 2008, Article ID 131294 (2008)

19. Ćirić, LB, Samet, B, Aydi, H, Vetro, C: Common fixed points of generalized contractions on partial metric spaces and an application. Appl. Math. Comput. 218, 2398-2406 (2011)

20. Di Bari, C, Vetro, P: Fixed points for $\varphi$-weak contractions on partial metric spaces. Int. J. Eng., Contemp. Math. Sci. 1, 5-13 (2011)

21. Dukić, D, Kadelburg, Z, Radenović, S: Fixed points of Geraghty-type mappings in various generalized metric spaces. Abstr. Appl. Anal. 2011, Article ID 561245 (2011)

22. Escardo, MH: PCF extended with real numbers. Theor. Comput. Sci. 162, 79-115 (1996)

23. Gopal, D, Imdad, M, Vetro, C, Hasan, M: Fixed point theory for cyclic weak $\phi$-contraction in fuzzy metric spaces. J. Nonlinear Analysis Appl. 2011, Article ID jnaa-00110 (2011)

24. Harjani, J, Sadarangani, K: Fixed point theorems for weakly contractive mappings in partially ordered sets. Nonlinear Anal. 71, 3403-3410 (2009)

25. Jungck, G: Compatible mappings and common fixed points. Int. J. Math. Math. Sci. 9, 771-779 (1986)

26. Karapinar, E: Fixed point theory for cyclic weak $\phi$-contraction. Appl. Math. Lett. 24, 822-825 (2011)

27. Kirk, WA, Srinivasan, PS, Veeramani, P: Fixed points for mappings satisfying cyclical contractive conditions. Fixed Point Theory 4, 79-89 (2003)

28. Lakshmikantham, V, Cirić, L: Coupled fixed point theorems for nonlinear contractions in partially ordered metric spaces. Nonlinear Anal. 70, 4341-4349 (2009)

29. Matthews, SG: Partial metric topology. In: Proceedings of the 8th Summer Conference on General Topology and Applications. Annals of the New York Academy of Sciences, vol. 728, pp. 183-197 (1994)

30. Nashine, HK, Samet, B: Fixed point results for mappings satisfying $(\psi, \varphi)$-weakly contractive condition in partially ordered metric spaces. Nonlinear Anal. 74, 2201-2209 (2011)

31. Nieto, JJ, Rodríguez-López, R: Contractive mapping theorems in partially ordered sets and applications to ordinary differential equations. Order 22, 223-239 (2005)

32. Nieto, JJ, López, RR: Existence and uniqueness of fixed point in partially ordered sets and applications to ordinary differential equations. Acta Math. Sin. Engl. Ser. 23(12), 2205-2212 (2007)

33. Oltra, S, Valero, O: Banach's fixed point theorem for partial metric spaces. Rend. Ist. Mat. Univ. Trieste 36(1-2), 17-26 (2004)

34. O'Regan, D, Petrusel, A: Fixed point theorems for generalized contractions in ordered metric spaces. J. Math. Anal. Appl. 341, 1241-1252 (2008)

35. Pacurar, M, Rus, IA: Fixed point theory for $\phi$-contractions. Nonlinear Anal. 72, 1181-1187 (2010)

36. Paesano, D, Vetro, P: Suzuki's type characterizations of completeness for partial metric spaces and fixed points for partially ordered metric spaces. Topol. Appl. 159, 911-920 (2012)

37. Radenović, S, Kadelburg, Z, Jandrlić, D, Jandrlić, A: Some results on weak contraction maps. Bull. Iranian Math. Soc. (to appear)

38. Ran, ACM, Reurings, MCB: A fixed point theorem in partially ordered sets and some applications to matrix equations. Proc. Am. Math. Soc. 132, 1435-1443 (2004)

39. Samet, B: Coupled fixed point theorems for a generalized Meir-Keeler contraction in partially ordered metric spaces. Nonlinear Anal. 72, 4508-4517 (2010) 
40. Samet, B, Rajović, M, Lazović, R, Stoiljković, R: Common fixed point results for nonlinear contractions in ordered partial metric spaces. Fixed Point Theory Appl. 2011, 71 (2011)

41. Sintunavarat, W, Cho, YJ, Kumam, P: Common fixed point theorems for $c$-distance in ordered cone metric spaces. Comput. Math. Appl. 62, 1969-1978 (2011)

doi:10.1186/1687-1812-2012-124

Cite this article as: Aydi et al:: Coincidence and fixed points for contractions and cyclical contractions in partial metric spaces. Fixed Point Theory and Applications 2012 2012:124.

Submit your manuscript to a SpringerOpen ${ }^{\circ}$ journal and benefit from:

- Convenient online submission

Rigorous peer review

- Immediate publication on acceptance

- Open access: articles freely available online

- High visibility within the field

- Retaining the copyright to your article

Submit your next manuscript at $\gg$ springeropen.com 